\title{
A Novel Carrier Phase Recovery for Optical Coherent Receivers with 16QAM Modulation Format
}

\author{
Shuhao Xue ${ }^{a}$, Xue Chen ${ }^{b}$, Wentao Liu ${ }^{c}$
}

State Key Lab of Information Photonics and Optical Communications, Beijing University of Posts and Telecommunications, P.O. Box 128, \#10 XiTuCheng Road, HaiDian District, Beijing 100876, China

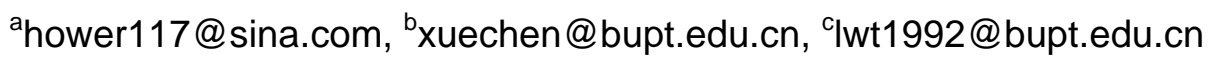

Keywords: Digital signal processing, Coherent receiver, optical, carrier phase recovery, quadrature amplitude modulation (QAM).

\begin{abstract}
We propose a novel carrier phase recovery algorithm based on constellation partitioning and maximum likelihood phase decision (MLPD) for square 16-ary quadrature amplitude modulation format in optical coherent transmission systems and verify its performance by PDM-16QAM simulation. Compared with classic carrier phase recovery algorithm like blind phase search algorithm, the simulation results show that the complexity of the proposed algorithm is much lower while the OSNR tolerance is very similar.
\end{abstract}

\section{Introduction}

High-order quadrature amplitude modulation (QAM) formats combined with coherent detection is a kind of advanced technologies that will be popular for future Optical transmission because of its high spectral efficiency and high data rate $[1,2,3]$. To recover the received signals, digital signal processing (DSP) has been widely applied. There are several algorithm modules in DSP for digital coherent receiver and the carrier phase recovery is a fundamental one $[4,5]$. Two types of phase estimation algorithm are used generally. One is feedback method, such as phase locked loop (PLL), and the other is feedforward method such as the $M$-th power algorithm and the blind phase search algorithm (BPS) $[6,7,8,9]$. Due to the high hardware requirement of PLL, feedforward phase estimation algorithm is preferred in most optical coherent receivers [10]. For $M$-QAM formats, when $M=4$, the classic $M$-th power phase recovery is usually applied. As the order of modulation formats increases like 16QAM, the estimation accuracy of $M$-th power algorithm would decrease, so BPS replaces it to play an important role to remove the phase offset owing to its high estimation accuracy and versatility. However, the problem associated with this method is its complexity: the required number of test phase angles increases with the modulation order. This problem has become a severe challenge for hardware in practical high-speed communication so an algorithm with low complexity is desired strongly for receiver DSP.

To reduce the algorithm complexity for high baud rate optical transmission, we propose a carrier phase recovery algorithm based on constellation partitioning and maximum likelihood phase decision (MLPD) for square 16QAM format, and demonstrate its performance for coherent optical communication system using polarization-division-multiplexed 16QAM (PDM-16QAM) by simulation. Compared with traditional BPS, our method could greatly lower the computational complexity. Since it could involve all the current symbols for phase estimation, the proposed method could get a performance close to BPS with small linewidth.

Principle. The section headings are in boldface capital and lowercase letters. Second level headings are typed as part of the succeeding paragraph (like the subsection heading of this paragraph). The block diagram of the proposed carrier phase recovery algorithm is given in Fig. 1(a). After clock recovery, dispersion equalization and frequency offset compensation, the $\mathrm{k}$-th received sample at the symbol rate could be expressed as $S(k)=A(k) \exp \left\{j\left[\theta_{S}(k)+\theta_{L}+\theta_{n}\right]\right\}$. A is the sample's amplitude; $\theta_{S}$ is the modulated phase of square 16QAM; $\theta_{L}$ is the phase error induced by laser linewidth, which could 
be regarded as the same in a short-time block; $\theta_{n}$ is the phase noise including ASE noise and device's electrical noise. To estimate the phase error $\theta_{L}$ more accurately, samples are recovered one by one using slide window whose block length is $\mathrm{N}$.

The proposed algorithm is based on constellation partitioning and maximum likelihood phase decision. Firstly, samples are partitioned into two subgroups, Class I (blank points) and Class II (solid points), as shown in Fig. 1(b). This operation is constellation partitioning. The Class I samples are characterized by their modulated phase angles of $\pi / 4+n \cdot \pi / 2(n=0 \ldots 3)$. It means the modulated phase of Class I samples could be limited among four phase values $\{\pi / 4,3 \pi / 4,5 \pi / 4,7 \pi / 4\}$. At beginning, samples are partitioned and the first sample $S_{1}$ which belongs to Class I in the block is selected as the reference value. Each sample in the same block is multiplied by the conjugation of the reference sample and the relative phase difference $\Delta \theta(k)$ between each sample and the reference could be obtained by calculating the angle of the product:

$$
\Delta \theta(k)=\arg \left[S(k) \cdot S_{1}^{*}\right] \approx \theta_{S}(k)-\theta_{S 1} .
$$

$\theta_{S 1}$ is the true modulated phase of the selected sample. It is noticed that there is no $\theta_{L}$ in the relative phase difference because of $\theta_{L}$ 's invariance.

Then the novel method is used to obtain one assumed modulated phase corresponding to one sample. This is based on maximum likelihood estimation: we could make a phase decision to get an assumed modulated phase according to the constellation region that the corresponding sample lies in as long as the sample is close to a reasonable ideal constellation point. This assumed modulated phase and the true one should be an integral multiple of $\pi / 2$ apart in phase so the phase difference could be removed by 4-th power operation. The mapping relationship is displayed in Fig. 1(c). It's obviously that Class I samples have better decision accuracy than Class II. We refer to this technique as the "maximum likelihood phase decision".

By using above method, the selected sample $S_{1}$ 's assumed modulated phase $\theta_{S 1}$ is assumed to be one value of $\{\pi / 4,3 \pi / 4,5 \pi / 4,7 \pi / 4\}$ because of the mapping relationship. The $k$-th sample in the block rotates to a new position and becomes $A(k) \cdot \exp \left[\theta_{S 1}+\Delta \theta(k)\right]$. Theoretically, when the reference sample is adjusted to a position nearby ideal constellation point's location, other samples also have corresponding approximate ideal positions respectively because the relative positions of these samples are fixed. Now each sample in the block could correspond to an assumed modulated phase $\theta_{D}(k)$. Then we multiply $S(k)$ by the conjugation of $S_{D}(k)$ to wipe off the true modulated phase from the whole phase of $S(k)$ :

$$
\begin{aligned}
S(k) \cdot S_{D}^{*}(k) & =A(k) \cdot \exp \left[j \theta_{S}(k)-j \theta_{D}(k)+j \theta_{L}+j \theta_{n}\right] \\
& =A(k) \cdot \exp \left[j\left(m \cdot \frac{\pi}{2}\right)+j \theta_{L}+j \theta_{n}\right], m=0, \ldots, 3 .
\end{aligned}
$$

where $S_{D}=\exp \left[j \theta_{D}(k)\right]$. The result is raised to 4 -th power to remove $m \cdot \pi / 2$, since $[\exp (j m \cdot \pi / 2)]^{4}=1$. After that the average phase error estimation for square 16QAM format could be extracted and the impact of $\theta_{n}$ could be mitigated by calculating the angle of the vector composed of the sum of the $\mathrm{N}$ normalized symbols as follow:

$$
\theta_{L, \text { est }}=\frac{1}{4} \arg \left\{\sum_{k}^{N} \frac{\left[S(k) \cdot S_{D}^{*}(k)\right]^{4}}{\left|\left[S(k) \cdot S_{D}^{*}(k)\right]^{4}\right|}\right\} .
$$



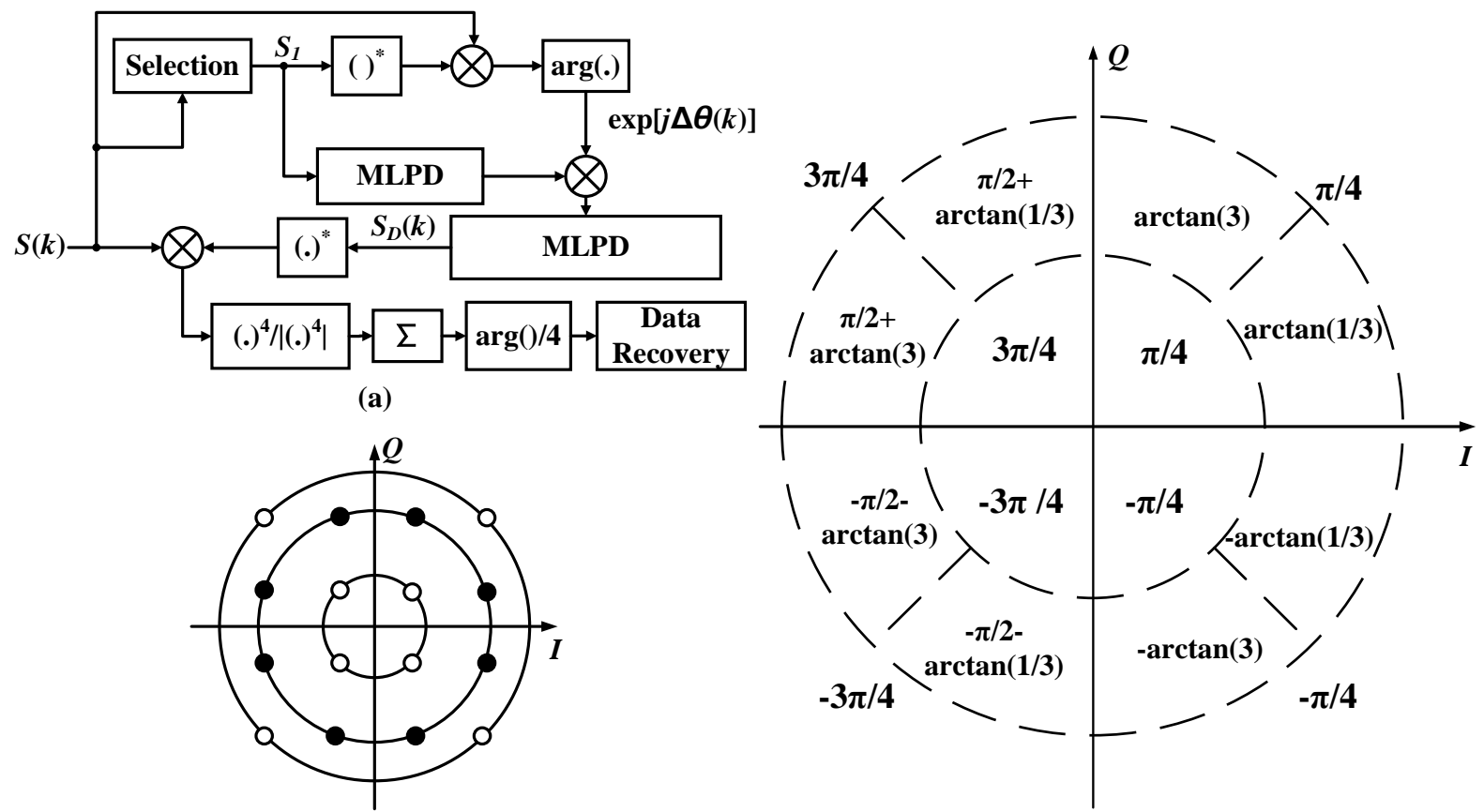

(b)

(c)

Fig. 1 (a) Principle of the proposed algorithm. (b) Class partitioning for 16QAM. (c) Maximum likelihood phase decision region for 16QAM.

Performance and verification by simulation. The performance of the proposed MLPD was evaluated by simulation based on VPI TransmissionMaker and Matlab. The simulation setup as showed in Fig. 2 was a 240Gb/s PDM-16QAM system using coherent receiver with various optical signal noise ratio (OSNR) and linewidth. At the transmitter side, optical square 16QAM signals are generated by driving optical IQ modulator with four 16QAM data sources $\left(I_{x}, Q_{x}, I_{y}, Q_{y}\right)$ and PDM is achieved by using polarizing beam splitter (PBS) and polarizing beam coupler (PBC). The receiver consists of the PBSs, local oscillator (LO), 90 degree hybrids, balanced photodetector (BPD) pairs and analog-digital converters (ADC). The linewidth of transmitter laser and local laser was set equally. The sampling rate of the receiver was $60 \mathrm{GSa} / \mathrm{s}$.

The sampled data were down sampled to one sample/symbol by DSP, where the optimum sampling phase was assumed. The average effective block sizes in both algorithms (the proposed one and BPS) were set to 20 for fair comparison. Effective block means all samples in the block involve in calculating.

Fig. 3(a) gives the bit error rate (BER) curves with different OSNR when laser linewidth was $100 \mathrm{kHz}$ in back-to-back (BtB) with zero frequency offset and in $10 \times 80 \mathrm{~km}$ transmission with $1 \mathrm{GHz}$ frequency offset. DSP was the same as showed in Fig. 2 DSP architecture except phase recovery method. Both methods have virtually the same performance especially with high OSNR under BtB and $10 \times 80 \mathrm{~km}$ respectively. The OSNR tolerance over $10 \times 80 \mathrm{~km}$ transmission is about $1 \mathrm{~dB}$ lower than the $\mathrm{BtB}$ expectation because of the transmission and residual frequency offset impairment. The results with ideal condition (BtB, zero laser linewidth and frequency offset) are displayed as a reference. It is shown that the penalties between $\mathrm{BtB}$ results and ideal condition results are small which means both algorithms have good phase compensation effects.

The results on linewidth impacts are shown in Fig. 3(b), where OSNR was $21 \mathrm{~dB}$ and frequency offset was zero. It is observed that the performance difference between two methods increases with the linewidth. It means the proposed algorithm is more sensitive to the noise phase than BPS under large linewidth because a larger linewidth induces a faster-changing symbol phase, resulting in that modulated phase may be decided by mistake, but the difference is little.

From the simulation, we find that if the proposed method only uses Class I symbols and increases the block size of carrier phase recovery to keep the number of effective samples same as before to remove the noise phase, the OSNR and linewidth tolerance could improve. The reason is that the area 
of assumed modulated phase decision region of Class I symbols is larger than the area of Class II so that the correct rate of assumed modulated phase decision is higher.
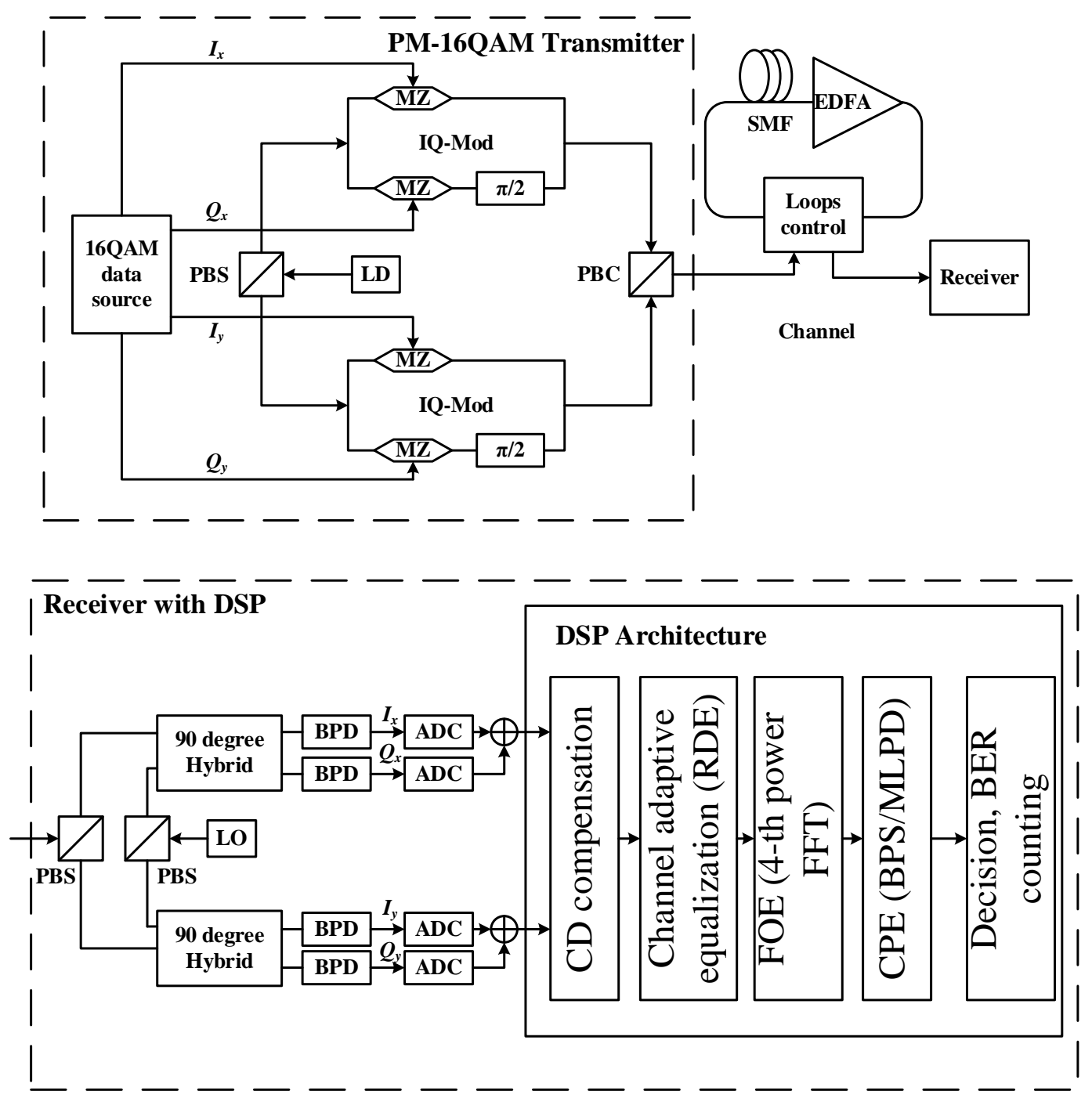

Fig. 2 The simulation setup

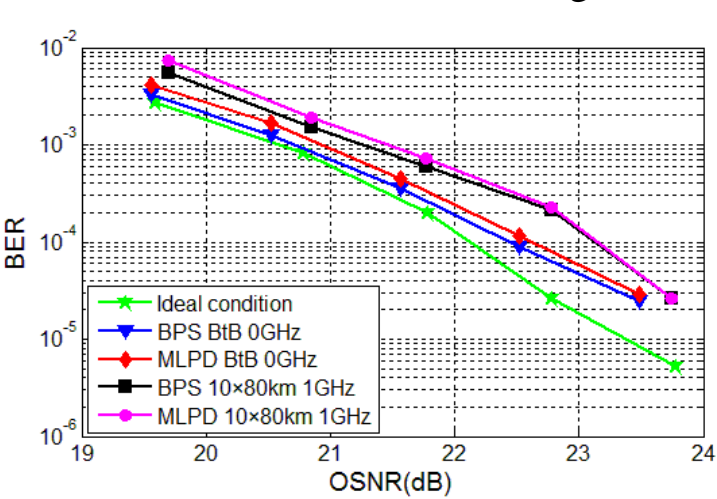

(a)

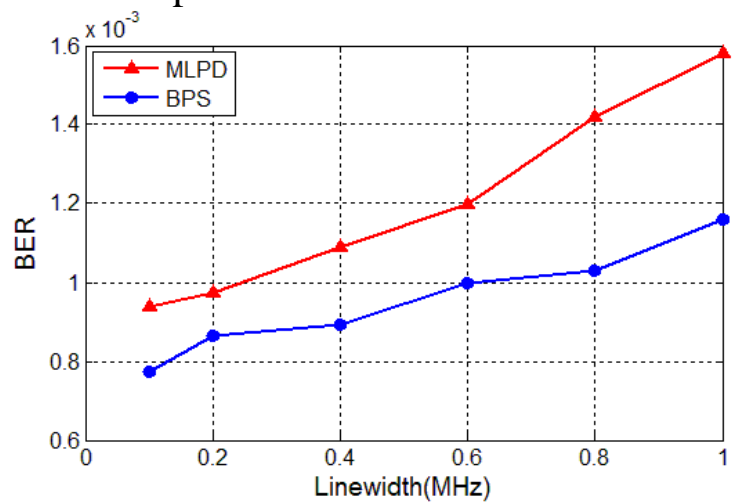

(b)

Fig. 3 (a) BER versus OSNR curves. (b) BER versus laser linewidth curves.

Table 1 gives the complexity per effective sample of proposed algorithm in comparison of the BPS algorithm in a 16QAM system. We discuss the required operation for an effective block and the effective block length is $N$. The complexity is measured by real number calculation. Here, the number of test phase of BPS is assumed as 32 for 16QAM.

It is clear that the proposed algorithm has obvious advantage in computational complexity, and the complexity is roughly 1/7 of BPS. In practice, the process could be simplified by only handling with the Class I symbols and ignoring the Class II symbols to reduce the algorithm complexity further at 
the expense of time delay. This simplified algorithm could be used for higher-order QAM formats like 64QAM and tolerate lower OSNR and larger linewidth.

Table 1 Complexity per effective sample of the proposed algorithm and BPS.

\begin{tabular}{lll}
\hline Algorithm & Real multiplier & Real adder \\
\hline MLPD & 20 & $12-2 / \mathrm{N}$ \\
BPS & 128 & $96+32 / \mathrm{N}$ \\
\hline
\end{tabular}

\section{Summary}

A novel digital carrier phase recovery algorithm for 16QAM modulation format with optical coherent receivers has been proposed. As compared to BPS, the proposed algorithm could achieve a similar performance under high OSNR and small linewidth $(<600 \mathrm{kHz})$ and reduce the DSP complexity greatly. Nowadays, a commercial laser with linewidth under $100 \mathrm{kHz}$ is available and usually used for coherent systems so this algorithm seems appropriate.

\section{Acknowledgements}

This study is supported by National Natural Science Foundation of China (No. 61571061) and Fund of State Key Laboratory of Information Photonics and Optical Communications (Beijing University of Posts and Telecommunications), P. R. China.

\section{References}

[1] G. Bennett, K. T. Wu, A. Malik, et al.: A review of high-speed coherent transmission technologies for long-haul DWDM transmission at 100G and beyond. Communications Magazine, IEEE Vol. 52 (2014), p. 102-110

[2] J. Geyer, CR. Doerr, M. Aydinlik, et al. in: Practical Implementation of Higher Order Modulation Beyond 16-QAM. Optical Fiber Communication Conference, paper Th1B. 1 (2015).

[3] E. Lach and W. Idler: Modulation Formats for 100G and Beyond. Optical Fiber Technology Vol. 17 (2011), p. 377-386

[4] A. Lau, et al.: Advanced DSP Techniques Enabling High Spectral Efficiency and Flexible Transmissions: Toward Elastic Optical Networks. IEEE Signal Processing Magazine Vol. 31 (2014), p. 82-92

[5] X. Zhou: Efficient Clock and Carrier Recovery Algorithms for Single-Carrier Coherent Optical Systems: A Systematic Review on Challenges and Recent Progress. IEEE Signal Processing Magazine Vol. 31 (2014), p. 35-45

[6] Y. Chen, Y. Pei and D M. W. Leenaerts, in: A Fully Integrated 30GHz 16-QAM Single-Channel Phased Array Transmitter with 5.9\% EVM at 6dB Back-off. European Solid-State Circuits Conference (ESSCIRC) -41st, pp. 92-95 (2015).

[7] A. J. Viterbi and A. M. Viterbi: Nonlinear Estimation of PSK-modulated Carrier Phase with Application to Burst Digital Transmission. Information Theory IEEE Transactions on Information Theory Vol. 29 (1983), p. 543-551

[8] T. Pfau, S.Hoffmann and R. Noé: Hardware-Efficient Coherent Digital Receiver Concept with Feedforward Carrier Recovery for M-QAM Constellations. Journal of Lightwave Technology Vol. 27 (2009), p. 989-999

[9] X. Zhou, C. Lu, A. P. T. Lau, et al.: Low-Complexity Carrier Phase Recovery for Square M-QAM Based on S-BPS Algorithm. IEEE Photonics Technology Letters Vol. 26 (2014), p. 1863-1866

[10]A. P. T.Lau, Y. Gao, Q. Sui, et al. in: Advanced DSP for High Spectral Efficiency and Flexible Optical Communications. Signal Processing in Photonic Communications, paper SM2D. 1 (2014). 